\title{
LA VERDAD EN EL GNOSTICISMO
}

\author{
THE TRUTH IN GNOSTICISM
}

Clayde Littlejohn

\begin{abstract}
RESUMEN
Hay dos supuestos sobre el valor epistémico que guían las discusiones más recientes sobre éste. El primero es que hay algo bueno con respecto a la creencia verdadera. El segundo supuesto es que es posible que dos creencias difieran en su valor incluso si ambas creencias son igualmente correctas. El veritista tiene fácil explicar el primer supuesto, pero tiene más difícil explicar el segundo. Para explicarlo, el veritista tiene que mostrar que las creencias verdaderas pueden diferir en su valor porque encarnan diferentes valores de bienes derivativos. El veritista tiene que proporcionarnos una historia de cómo es posible que pares de creencias verdaderas difieran en su valor epistémico no-instrumental. La forma más prometedora que una explicación de este tipo puede tomar, en mi opinión, apela al trabajo de Sosa (2007) sobre el valor epistémico y la naturaleza del conocimiento. Según la postura de Sosa, el conocimiento es un tipo de logro, un tipo de éxito que es atribuible al sujeto y a sus habilidades. Estos logros, nos sugiere, son más valiosos que un caso de éxito atribuible a la suerte y que no constituye un logro. Presentaré algunas dificultades potenciales para su postura y consideraré si tiene los recursos para lidiar con ellas. Voy a delinear los detalles de una postura alternativa que evade estas dificultades. Tal postura retiene el análisis del conocimiento de Sosa, pero requiere que revisemos algunas propuestas veritistas sobre el valor epistémico. La postura revisada nos proporciona algunas herramientas que serán útiles al tratar de ofrecer una teoría de la creencia racional o diestra.
\end{abstract}

PALABRAS CLAVE: Ernest Sosa, veritismo, creencia diestra, valor epistémico

\begin{abstract}
Two assumptions about epistemic value guide most recent discussions of it. The first is that there is something good about true belief. The second assumption is that it's possible for two beliefs to differ in value even if both beliefs are equally accurate. The first assumption about value is easy to explain on the veritist view. But veritists have a harder time explaining the second assumption. To explain it, the veritist needs to show that true beliefs can differ in value because they realize different derivative goods or values. What the veritist needs to tell us is some story about how it's possible
\end{abstract}


for pairs of true beliefs to differ in their non-instrumental epistemic value. The most promising form such an explanation can take, in my view, draws on Sosa's (2007) work on epistemic value and the nature of knowledge. On Sosa's view, knowledge is a kind of achievement, a success that is attributable to the subject and her abilities. These achievements, he suggests, are more valuable than a success that is down to luck and does not constitute an achievement. I'll raise some potential difficulties for his view and consider whether he has the resources to address them. I shall outline the details of an alternative view that avoids these difficulties. It retains Sosa's account of knowledge but requires us to revise some veritist proposals about epistemic value. The revised view provides us with some tools that will be useful in trying to offer a theory of adroit or rational belief.

KEYWORDS: Ernest Sosa, Veritism, adroit belief, epistemic value.

\section{INTRODUCCIÓN}

Hay dos supuestos sobre el valor epistémico que guían las discusiones más recientes sobre éste. ${ }^{1}$ El primero es que hay algo bueno con respecto a la creencia verdadera. ${ }^{2}$ Las intuiciones que dan soporte a éste han sido expresadas de distintas maneras. Algunos autores (e.g., Zabzebski (1996)) parecen defender la postura de que el estado de cosas en el que Agnes cree una proposición verdadera es uno bueno, quizás uno que podría ser bueno para ella. Otros autores (e.g., Sosa (2007)) no dicen esto y discuten en su lugar la idea de que la verdad es un valor fundamental en un tipo de dominio evaluativo específico. Se deja abierta la cuestión de si alcanzar estos bienes vuelve al mundo un mejor lugar para vivir o si hace que nuestras vidas mejoren. La idea esencial parece ser que las creencias verdaderas tienen, todas ellas, una virtud y ésta es una virtud que ninguna creencia falsa podría tener. El

\footnotetext{
${ }^{1}$ Me gustaría dar las gracias a María Álvarez, Thomas Byrne, Charles Cote-Couchard, Peter Dennis, Julien Dutant, David Owens, David Papineau y Barnaby Walker por las útiles discusiones.

${ }^{2}$ Ésta es una intuición que figura prominentemente en las discusiones del valor epistémico de Goldman (1999), Pritchard (de próxima aparición), Sylvan (2013), y Zazebski (1996). Es difícil determinar cuál es la opinión de Sosa con respecto a este supuesto, pero hay pasajes en los que parecería que tiene cierta simpatía hacia este. Podríamos leer la discusión de Sosa (2007) sobre la normatividad epistémica como un desarrollo de esta idea, pero esta lectura es de alguna manera especulativa y podríamos leer este trabajo como si explorase las consecuencias de 'el monismo epistémico de la verdad’ sin adoptar tal postura. En su (2001), dice explícitamente que este monismo es sólo un supuesto.
} 
segundo supuesto es que es posible que dos creencias difieran en su valor incluso si ambas creencias son igualmente correctas. ${ }^{3} \mathrm{Si}$, digamos, Agnes sabe que tienes que dar vuelta a la izquierda para llegar a Larissa y yo creo, pero no sé, que esto es así, la creencia de Agnes es comparativamente mejor que la mía. De manera similar, si las creencias de Agnes con respecto a cómo es Larisa durante esta época del año son correctas pero no están justificadas, mis creencias sobre el clima de Larissa podrían ser mejores que las suyas si mis creencias están justificadas.

El veritista piensa que hay uno y sólo un bien epistémico fundamental y que este bien es la creencia verdadera. Nuestro primer supuesto acerca del valor es fácil de explicar desde el punto de vista veritista. El veritista piensa que todas y sólo las creencias verdaderas encarnan el bien epistémico fundamental. Pero el veritista tiene más dificultades explicando el segundo supuesto. Para explicarlo, el veritista tiene que mostrar que las creencias verdaderas pueden diferir en su valor porque encarnan diferentes valores de bienes derivativos.

Es fácil pensar en muchas maneras distintas en las que las creencias podrían diferir en su valor. A Charles le gusta recordarme que la leche es la bebida estatal de Delaware. A mí me gusta recordarle que éste es el hecho menos interesante del mundo. A diferencia del hecho de Charles, éste es interesante. Nuestras creencias difieren en términos de qué tan interesantes son, pero no es muy útil señalar tales diferencias porque (a) no es claro que la diferencia entre interesante y no interesante sea una que importa para el dominio evaluativo relevante y (b) esta diferencia no empata con las diferencias que estamos tratando de entender (e.g., la diferencia en valor que explica por qué es mejor saber que $p$ o creer justificadamente que $p$, que simplemente tener una creencia correcta sobre $p$ ). Como Firth (1981) y Pascal nos recuerdan, algunas creencias difieren en términos de su valor instrumental. A pesar de que esto es interesante, es difícil ver cómo es que esto podría ser relevante dados nuestros intereses, porque no pensamos que el conocimiento sea mejor que la mera creencia verdadera debido a su potencial diferencia en términos de su valor instrumental. El veritista tiene que proporcionarnos una historia de cómo es posible que pares de creencias verdaderas difieran en su valor epistémico no-instrumental.

La forma más prometedora que una explicación de este tipo puede tomar, en mi opinión, apela al trabajo de Sosa (2007) sobre el valor epistémico y la naturaleza del conocimiento. Según la postura de Sosa, el conocimiento es un tipo de

\footnotetext{
${ }^{3}$ Para una discusión crítica de esta intuición véanse Dutant (2012), Pritchard (de próxima aparición), y Sartwell (1992).
} 
logro, un tipo de éxito que es atribuible al sujeto y a sus habilidades. Estos logros, nos sugiere, son más valiosos que un caso de éxito atribuible a la suerte y que no constituye un logro. Nuestra discusión comienza con un tratamiento del problema del Menón que apela al trabajo se Sosa. Con esto en mente, presentaré algunas dificultades potenciales para su postura y consideraré si tiene los recursos para lidiar con ellas. Voy a delinear los detalles de una postura alternativa que evade estas dificultades. Tal postura retiene el análisis del conocimiento de Sosa, pero requiere que revisemos algunas propuestas veritistas sobre el valor epistémico. La postura revisada nos proporciona algunas herramientas que serán útiles al tratar de ofrecer una teoría de la creencia racional o diestra.

\section{LA CREENCIA VERDADERA, LA QUE ES MEJOR, Y LA QUE ES APTA}

La mayoría de las discusiones del valor epistémico operan bajo dos supuestos:

A1: Las creencias verdaderas son epistémicamente buenas en tanto que poseen una característica que las vuelve buenas, una característica que ninguna creencia falsa posee.

A2: Los pares de creencias verdaderas pueden diferir en sus propiedades evaluativas epistémicas de manera tal que una posea una característica que la vuelve buena, de la cual la otra carezca (e.g., una creencia verdadera y justificada sobre $p$ es mejor que una creencia verdadera sobre $p$, saber que $p$ es mejor que creer que $p$, etc.).

Es fácil ver cómo un veritista podría dar cuenta de (A1), pero ¿cómo podría dar cuenta de (A2)? Consideremos algunos pares de creencias específicos, tales como mi creencia acerca del camino a Larisa y la creencia de Agnes al respecto. Podemos estipular que nuestras creencias son igualmente correctas pero que difieren sólo en que Agnes sabe que hay que dar vuelta a la izquierda. Esto nos debería de dar un par específico en el que una creencia correcta es mejor que la otra. Si queremos entender por qué este par difiere en su valor, deberíamos comenzar considerando dos partes de la postura epistemológica de Sosa, su teoría del conocimiento y su teoría del valor epistémico.

De acuerdo con la teoría del conocimiento de Sosa, la creencia de Agnes constituye conocimiento porque la corrección de su creencia es atribuible a su habilidad. El conocimiento, de acuerdo con esta postura, es un tipo de logro. Claramente, Agnes y yo podemos, ambos, tener creencias verdaderas sobre el camino a Larissa, pero hay algo acerca de la manera en la que la creencia de Agnes ha sido formada que nos llevaría a decir que la corrección de su creencia es atribuible a ella y a la manera en la que ejercitó sus capacidades racionales, mientras que no 
diríamos algo similar con respecto a mi creencia y mis habilidades. Su creencia es conocimiento porque es correcta debido a su destreza. Mi creencia no es conocimiento porque es correcta sin serlo debido a mi destreza.

¿Cómo nos ayuda esto a entender (A2)? Si asumimos, como lo haremos en esta discusión, que las creencias verdaderas o correctas constituyen conocimiento en virtud de que son un tipo específico de logro, uno en el que la corrección es atribuible a la habilidad, obtenemos la explicación que buscamos si añadimos el supuesto adicional de que los logros son valiosos de ciertas maneras que los meros resultados afortunados no lo son. Si un éxito es un logro, es mejor que un éxito que no puede ser pensado como si fuera ningún tipo de logro.

La idea que nos guía aquí es bastante simple. Cuando consideramos pares de individuos que, digamos, tratan de hacer lo mismo (e.g., hacer una taza de café, mantener un registro correcto de ciertos eventos, o asar un cerdo), algo bueno puede resultar si estos individuos son lo suficientemente afortunados para tener éxito en sus empresas, pero es mejor tener éxito en estas empresas gracias a su habilidad que simplemente ser lo suficientemente afortunado para tener éxito en la empresa relevante. El valor de la actuación excelente deriva del bien alcanzado por el éxito de la empresa, de manera que la teoría es compatible con el tipo de supuesto monista que los veritistas adoptan, pero el valor de la actuación excelente no se reduce al valor alcanzado mediante el éxito de la empresa. ${ }^{4}$

De acuerdo con este marco general del valor, entonces, podemos pensar en un tipo de bien básico, como el bien que se consigue al alcanzar la meta (la verdad (quizás) en el caso de la creencia, o precisión en el caso de lanzamiento de herraduras) y otro tipo de bien derivativo, como el bien que se consigue siempre y cuando alcanzar la meta sea atribuible al excelente uso de las habilidades de un individuo. Dado que hemos identificado el conocimiento con un tipo de logro, hemos explicado por qué el par relevante de creencias verdaderas puede diferir en valor, de una manera que es consistente con (A1) y con el veritismo. ${ }^{5}$ En la je-

\footnotetext{
${ }^{4}$ Para una versión interesante de esta cuestión, una que maneja bien la objeción del empantanamiento [nota del traductor: swamping objection], véase Sylvan (2013).

${ }^{5}$ Es difícil decir si Sosa acepta las posturas veritistas estándar. Para empezar, está en desacuerdo con veritistas como Kvanvig (2003) y Lynch (2004) con respecto al valor de verdades triviales y no interesantes. Por otro lado, hay pasajes en los que sugiere que la creencia apta o el conocimiento son ellos mismos un valor fundamental, quizás adicionales al valor de la creencia verdadera (Sosa 2007: 88). Hay también pasajes en los que considera que la meta de la creencia y del juicio es la creencia apta o el conocimiento. (Véase la discusión de Sosa (2015: 55) sobre la adivinación y el juicio). Esto podría sugerir que la creencia apta o el conocimiento (animal)
} 
rarquía, la creencia verdadera o correcta ocupa el papel que ocupan los éxitos y el conocimiento ocupa el rol de los éxitos atribuibles a la habilidad. Hay que recordar que puede haber desacuerdos con respecto a las cosas que ocupan estos papeles, desacuerdos que son de alguna manera ortogonales a los debates con respecto a si los éxitos que surgen de la habilidad son mejores que los éxitos que meramente se deben a la suerte o a la buena fortuna. Podemos llamar a estos los supuestos substantivo y estructural, respectivamente.

Esta teoría hace un buen trabajo lidiando con un desafío relacionado en el problema del Menón. Si elegimos un par de creencias diferente, por ejemplo una creencia que es verdadera pero que no está justificada y una que es verdadera y que está justificada, podríamos de nuevo pensar que hay una diferencia importante en el valor de estas creencias, una diferencia que nuestra teoría del valor debería explicar. Si la creencia relevante no es un caso de éxito atribuible a la habilidad, podría sin embargo ser un caso en el que las habilidades del sujeto fueron ejercitadas de manera apropiada. Así, esto podría explicar el hecho de que es mejor tener una creencia verdadera que está justificada que una que no lo está. En tales casos, podríamos dar cuenta de (A2) apelando a la destreza en lugar de a la aptitud.

Los críticos han señalado problemas potenciales con esta teoría, pero ninguna de las críticas que hemos visto hasta ahora me parecen fatales. ${ }^{6}$ En la siguiente sección presentaré dos desafíos que no han sido discutidos ampliamente en la literatura para determinar si la postura de Sosa puede lidiar con éstos.

\section{DOS DESAFÍOS}

En las discusiones sobre el valor epistémico parece haber un tercer supuesto que es aceptado de manera extendida sin recibir mucha atención en absoluto:

A3. Las creencias que son buenas epistémicamente son distintivamente valiosas.

deberían desplazar a las creencias verdaderas como el bien fundamental en la jerarquía, pero en (2015) también sugiere que hay estados mentales que buscan sólo la verdad que puede constituir conocimiento animal, así que no abandona de maneta total la idea de que la verdad podría ser un tipo de éxito.

${ }^{6}$ El desafío más serio, desde mi punto de vista, viene de la discusión de Hackner (20013) acerca de la relación que hay entre el conocimiento y los logros, pero estoy dejando de lado estas preocupaciones dado el propósito de nuestra discusión. Tengo simpatía por la sugerencia de Hackner de que una actitud apta no es necesariamente un logro. Para una respuesta útil a las preocupaciones acerca de los logros, sin embargo, véase Ho (de próxima aparición). Véase Bradford (2015) para una excelente guía a la naturaleza y valor de los logros en general. 
La idea detrás de (A3) es bastante simple. Una creencia no es sólo potencialmente valiosa, tiene el potencial de ser valiosas de ciertas maneras que una no-creencia no lo puede ser. ${ }^{7} \mathrm{Si}$ (A3) fuese falsa, debería haber estados o eventos mentales no-doxásticos que podrían hacer a la creencia superflua. Podríamos obtener todos los bienes asociados con la creencia buena sin tener la creencia. Sería entonces un misterio por qué debería importar si tenemos creencias en absoluto dado que podríamos alcanzar los mismos bienes sin ellas.

Si seguimos la iniciativa de Sosa pareciera que las propiedades que las creencias epistémicamente deseables tendrían son las siguientes. Pueden ser correctas, pueden ser diestras, y pueden ser aptas. Es fácil entender en qué consiste la corrección. Una creencia es correcta si y sólo si es una creencia verdadera. Una creencia será diestra si es producida mediante el ejercicio del tipo correcto de habilidades. Sosa caracteriza estas habilidades haciendo referencia a la meta de la corrección. Una creencia será apta si es correcta debido a la destreza. Explicar con detalle esta idea es difícil, pero la idea es que la corrección de alguna manera manifiesta la habilidad, de manera tal que atribuimos la corrección al agente o a sus habilidades cognitivas relevantes. Esto requiere más que el simple hecho de que las habilidades sean ejercitadas y que esto tenga como resultado una creencia correcta, de la misma manera que la digestión requiere más que el simple hecho de que te llenes con sobras de comida y que después ésta encuentre el camino a tus células.

Hay una preocupación sobre esta postura general. De acuerdo con muchas concepciones de la experiencia perceptiva, la experiencia es similar a la creencia en que puede ser tanto correcta como apta. ${ }^{8}$ En casos de ilusión, algunos filósofos sugieren, la experiencia se considera incorrecta. En casos de alucinación verídica, añaden, se la considera correcta incluso cuando la corrección de la experiencia no es atribuible a ninguna habilidad. Una vez que tenemos estas posibilidades en la mesa, parece que debería ser posible que haya un tipo de destreza que puede estar presente o ausente en casos de percepción. Si te parece visualmente que un objeto visible es F y la representación de este objeto en la visión como si fuese F es cau-

\footnotetext{
${ }^{7}$ El tipo de creencia que tengo en mente es la creencia plena o la creencia binaria. Algunos filósofos piensan que la creencia binaria podría no traer nada interesante a la discusión que no venga ya con la creencia parcial, pero veremos más adelante una razón para pensar que sí lo hace.

${ }^{8}$ Vénase Byrne (2009), Logue (2014), McDowell (1994), Schellenberg (2014) y Siegel (2011) para defensas de teorías representacionales de la experiencia perceptiva. Para argumentos en contra, véanse Brewer (2011) y Travis (2004). Yo estoy convencido por los argumentos en contra.
} 
sada por el objeto incorrecto, tendríamos corrección sin aptitud. Si es necesario presentar un ejemplo, los casos más simples son casos de experiencia visual que representan algo como si instanciaran alguna propiedad de alto nivel. Al ver un perro, alguien que piensa que la experiencia puede involucrar la presentación de propiedades de alto nivel podría decir que un niño puede ver al perro como peligroso. Si esa representación es provocada, digamos, precisamente por las características distintivas de los perros, de manera que el niño está predispuesto a ver a todo perro como peligroso, podemos imaginar casos en los que le presentamos al niño un perro asesino, lindo, abrazable y esponjoso. El perro podría ser peligroso, pero no sería visto de esa manera por nadie que supiese algo acerca de los perros, puesto que no presenta ninguna de las propiedades que indicarían que es peligroso. Aquí la experiencia del niño sería correcta, pero no sería apta ni diestra. La percepción apta, si hay tal cosa, requiere que las representaciones sean provocadas por el tipo correcto de cosas y esto sugiere, a su vez, que hay un papel para la destreza al entender la diferencia entre representación visual apta y meramente correcta.

En resumen, muchas posturas de la experiencia perceptiva implican que las experiencias podrían ser como las creencias en el sentido de que pueden ser aptas (i.e., correctas debido a la habilidad) y no meramente correctas. Podemos conceder que no decimos que las experiencias pueden ser conocimiento, pero esto no importa aquí. Un estado de conocimiento es, en la postura de Sosa, bueno porque es apto. De eta manera, la observación de que tanto la experiencia como la experiencia visual pueden ser aptos apunta a un problema real para (A3). Las características que hacen bueno al conocimiento, que explican su valor y que explican por qué el conocimiento es mejor que la creencia verdadera, son comunes tanto al conocimiento como a algunas formas de percepción.

El segundo desafío tiene que ver con el tratamiento veritista de los casos Gettier. Si compartes las intuiciones que prestan apoyo a (A1) y (A2) y consideras que la presencia/ausencia de conocimiento es algo que asegura una diferencia correspondiente en valor epistémico, entonces ciertamente puedes usar la teoría del valor de Sosa para explicar por qué es mejor saber que $p$ que simplemente creer que $p$ cuando $p$ es verdadera. Hay un problema adicional que los casos Gettier causan, y no es el desafío de explicar (A2), sino de defender (A1). Los casos Gettier pueden utilizarse para desafiar precisamente las intuiciones que prestan apoyo a (A1).

Veamos estos dos desafíos con mayor detalle. Comenzaremos con el desafío de la suficiencia y después consideraremos el desafío de la distinción. 


\subsection{El desafío de la suficiencia}

Se reconoce de manera general por parte de quienes favorecen el veritismo que no valoramos a la verdad, per se. Damos valor sólo a cosas como poseer la verdad, estar en contacto con la verdad, o seguir la pista de la realidad:

Al amar la verdad, entonces, lo que valoramos no es que las verdades sean verdaderas. Lo que valoramos al buscar la verdad es en su lugar su aprehensión, o que poseamos la verdad. ¿Qué significa esto? Sólo al creerla uno posee de manera relevante la verdad: poseemos la verdad de que la nieve es blanca al creer que la nieve es blanca. Al buscar la verdad lo que queremos es (al menos) creencias verdaderas (Sosa 2001: 49).

Esto es considerado por algunos escritores como si apoyara la postura de que "es bueno creer lo que es verdadero" (Lynch 2004: 12). En mi lectura, Lynch parece sugerir que toda creencia verdadera encarna algún tipo de valor. Podemos llamar esta tesis la tesis de la suficiencia.

La intuición de que hay algo bueno con respecto a poseer la verdad sólo presta apoyo a (A1) bajo el supuesto de que poseer una creencia verdadera es, inter alia, poseer la verdad. Soy escéptico sobre esto. Consideremos la noción de seguirle la pista a la realidad o de mantenerse en contacto con la realidad. Si pensamos que estas cosas son básicamente equivalentes a poseer la verdad, podríamos preguntar si es verdad que un sujeto está en contacto con la realidad si y sólo si tienen una creencia verdadera. Cuando pensamos acerca de las distintas maneras en las que un sujeto podría tener una creencia verdadera sin tener conocimiento, pareciera que la creencia verdadera no es suficiente para estar en contacto con la realidad precisamente porque la creencia verdadera no es suficiente para el conocimiento.

Ésta es una de las lecciones que deberíamos extraer de los casos Gettier. Consideremos una variación de la máquina de experiencias que nos presenta Nozick. ${ }^{9}$ La máquina induce experiencias que llevan de manera predecible a Agnes a creer varias cosas sobre el mundo externo. Es parte de la historia que las experiencias que la máquina produce no son producidas por lo que ocurre en los alrededores de Agnes, de manera que hay un sentido en el que ha perdido el contacto con una parte importante de la realidad al conectarse a la máquina. No recupera el contacto si, debido al tipo de suerte que encontramos en los casos Gettier, sucede que tiene una creencia verdadera. Podría creer, de manera correcta, que su hermana

\footnotetext{
${ }^{9}$ Véase Hyman (2015) para puntos relacionados sobre la relación entre el conocimiento y las razones.
} 
se encuentra bien porque cree, de manera correcta nuevamente, que su hermana acaba de entregar su tesis. Si tales creencias son producidas en la máquina de experiencias porque los programadores creen que las alucinaciones que inducen estas creencias harían feliz a Agnes, ella no está en contacto con los hechos sólo porque esta coincidencia increíble ocurre con respecto a cómo son las cosas miles de millas más allá de la máquina y de cómo son las cosas en el engaño de Agnes.

Si adoptamos el veritismo y aceptamos (A1), tenemos que decir que hay algo bueno con respecto a las creencias de Agnes, pero soy escéptico con respecto a que haya algo particularmente bueno acerca de la corrección o la verdad cuando lidiamos con casos en los que el creyente no se encuentra en contacto con las partes relevantes de la realidad. ${ }^{10}$ Una vez que somos escépticos con respecto a (A1), deberíamos ser escépticos de igual manera de las posturas que pretenden explicar (A2) proponiendo comenzar con el supuesto de la tesis de la suficiencia y tratar de mostrar que hay algún valor epistémico derivativo que explica por qué algunas creencias son mejores que otras. Una explicación alternativa, una que yo prefiero, explica (A2) mostrando que algunas creencias verdaderas son mejores que otras mostrando que algunas son buenas y algunas son o bien malas o inútiles.

Para entender la motivación de este tipo de postura podemos comenzar preguntándonos por qué valoramos la verdad. ¿Hay algo que pueda decir el veritista que explique su amor por la verdad? La explicación que yo ofrecería, si alguien me preguntase, sería que hay que empezar por la idea, presentada en la sección previa, de que una elucidación completa del valor que encarnan las creencias apelaría a alguna característica distintiva de las creencias. Las dos cosas que vienen a la mente son éstas. La creencia es única en la medida que tiene el potencial de ser conocimiento. La creencia es única en que tiene el potencial de proveernos con razones especificadas proposicionalmente, ${ }^{11}$ razones que son potenciales razones que motivan. Estos dos puntos están vinculados si, como sugerí antes, la creencia de que $p$ pone al sujeto en una posición para hacer $\Phi$ por la razón de que $p$ si y sólo si el sujeto sabe que $p$ o cree de manera apta que $p$.

\footnotetext{
${ }^{10}$ En lugar de (A1), defendería (A1*): las creencias falsas son epistémicamente malas en tanto que poseen una característica que las vuelve malas, una característica de la cual carecen algunas creencias verdaderas. Sosteniendo el gnosticismo, me gustaría decir que las únicas creencias que carecen de este defecto son aquellas que constituyen conocimiento.

${ }^{11}$ Para una discusión útil de las razones en general y una defensa de la postura de que las razones que motivan son hechos, véase Alvarez (2010). Para argumentos a favor de la posibilidad de razones falsas, véase Comesaña y McGrath (2014).
} 
Comenzando con esta idea, podemos argumentar en contra de la tesis de la suficiencia de la siguiente manera. El bien fundamental que puede encarnar una creencia es obtenido si y sólo si la creencia pone una verdad en nuestra posesión en el sentido de que nos permite hacer $\Phi$ por la razón de que $p$. Si bien es cierto que ninguna creencia falsa podría hacer esto (porque todas las razones especificadas proposicionalmente son verdaderas), no toda creencia verdadera podría hacerlo. Si un sujeto cree que $p$ sin saber que $p$, su creencia no lo pone en una posición para hacer $\Phi$ por la razón de que $p$ porque eso requiere que el hecho de que $p$ pueda guiar de manera racional al sujeto al hacer $\Phi$ y esto requiere un tipo de conexión no-accidental con el hecho que, de esta manera, requiere conocimiento.

Un argumento en contra de la tesis de suficiencia es un argumento en contra del veritismo dado que el veritismo cree que la verdad de una creencia es necesaria y suficiente para la realización del bien epistémico fundamental. La postura alternativa que ofrecería en lugar del veritismo es el gnosticismo, la postura de que hay uno y sólo un bien epistémico fundamental y que ese bien es el conocimiento. ${ }^{12}$ De acuerdo con esta postura, las creencias que encarnan el bien epistémico fundamental son las que constituyen conocimiento. Una vez que vemos por qué alguien podría adoptar esta postura, podemos comenzar a ver por qué algunas personas han sido engañadas para aceptar el veritismo. Hay algo bueno sobre poseer la verdad y con estar en contacto con los hechos de manera tal que puedas ser guiado por ellos, pero esto sólo parece apoyar al veritismo cuando no tomamos en cuenta el hecho de que poseer los hechos involucra más que tener una creencia verdadera. Una vez que vemos que (A1) es de hecho falsa, podemos ver que es mucho más fácil explicar (A2) de lo que los veritistas han pensado. Hay algo bueno sobre el conocimiento y nada bueno sobre creer sin saber. Cualquier creencia que no constituye conocimiento es un mal estado para el sujeto puesto que tal estado pretende ponerte en contacto con ciertas realidades cuando en realidad no lo hace. Al estar en tal estado, eres engañado por el pensamiento de que los hechos pueden guiarte cuando de hecho los hechos no están a tu alcance.

\footnotetext{
${ }^{12}$ Los lectores interesados en los evangelios gnósticos deben consultar Hyman (2015) y Williamson (2000). Debido a ciertos pensamientos confusos sobre casos de graneros falsos y experiencias visuales proposicionales, rechacé el gnosticismo en mi (2012), pero he visto la luz ahora y he intentado corregir mis errores pasados sobre graneros falsos en mi (2014). Debo el término "gnosticismo" a Margot Strohminger. Matt Benton sugirió "conosticismo" como una alternativa. En mi opinión, éste queda en un cercano segundo lugar.
} 


\subsection{E1 desafío de la distinción}

Para resolver o disolver el problema de la distinción debemos o bien desafiar el supuesto de que las experiencias perceptivas pueden ser aptas o bien identificar alguna característica de la creencia que la distinga de la experiencia perceptiva y que nos ayude a explicar (A3). Si bien creo que las experiencias perceptivas no son representacionales, no creo que encontraremos una respuesta satisfactoria al desafío de la distinción argumentando que las experiencias perceptivas podrían no ser aptas. No creo que haya una razón particularmente buena para negar que podría haber creaturas que posean estados no-doxásticos evaluables en relación a su corrección, que podrían ser potencialmente correctos debido a la destreza. Quizás podría haber o podría haber habido apariencias proposicionales (las cosas que los conservadores fenoménicos llaman "apariencias"). ${ }^{13}$ Si tales cosas son posibles, algunas de ellas podrían ser aptas y ninguna de ellas podría ser una creencia. El desafío permanecería incluso si rechazamos las teorías representacionales de la experiencia perceptiva.

Una estrategia más prometedora sería identificar una característica distintiva de la creencia, algo que la distinga de otros estados mentales representacionales potencialmente aptos. Una sugerencia que surge del trabajo de Sosa (2013, 2015) sobre la creencia y el juicio sería comenzar con la idea de que la creencia o el juicio podrían considerarse actuaciones. La experiencia perceptiva es una pasión, algo de lo que el sujeto que percibe sufre. Mientras que una experiencia perceptiva o una apariencia podrían ser aptas, ninguna de ellas podría ser una actuación excelente por parte de un agente.

$\mathrm{Al}$ introducir la agencia en la discusión podríamos argumentar que la presencia de la agencia es la característica adicional que explica por qué, digamos, la creencia apta es mejor que la experiencia apta, o bien podríamos argumentar que la característica adicional es ella misma una condición necesaria para la realización de algún tipo de valor. Para tener una idea del contraste, consideremos una línea de argumento familiar con respecto al valor moral. Consideramos a algunas acciones que tienen valor moral como si fuesen mejores que aquellas que meramente son hechas de manera correcta o que están permitidas. Podríamos ver los factores que distinguen a la acción correcta que tiene valor de la acción meramente correcta como algo que trae valor adicional a la mesa. Consideremos, en su lugar, una evaluación de la acción humana y animal. La vaca y el granjero pueden llevar a cabo acciones que son óptimas, pero presumiblemente sólo las acciones del granjero pueden

${ }^{13}$ Véase Huemer (2007), Tucker (2010), y Reiland (2015). 
contar como moralmente correctas. La presencia de cierto tipo de agencia podría considerarse como una precondición para la realización de un tipo de valor (i.e. corrección) pero no una característica que añade valor a aquello que realiza el valor.

Una dificultad que enfrentamos si tratamos de explicar (A3) apelando a la creencia o a la relación del juicio con la agencia es que distinguimos creencia apta de percepción apta pero no parecemos hacer un buen trabajo distinguiendo la creencia apta de la adivinación apta. Removemos un problema para (A3), pero surge uno nuevo. Una adivinación, como una creencia, puede evaluarse en términos de corrección. Algunas adivinaciones son mejores que otras y nos gustaría decir que algunas adivinaciones son diestras mientras que otras no lo son. Algunas adivinaciones podrían ser correctas debido a la destreza, así que parece que podría haber adivinaciones aptas. Aún más, parece que una adivinación podría involucrar agencia de todas las maneras que la creencia y el juicio lo hacen, de modo que no querríamos decir que nuestra agencia no está involucrada en la adivinación. Si consideramos a las adivinaciones y a los juicios o a las creencias como si difirieran en tipo y no consideramos a la posible adivinación buena como una amenaza a (A3), me parece que la agencia puede ser sólo parte de la explicación de (A3).

En lugar de pensar en una característica distintiva que distinga a algunas actitudes de otras (e.g., la relación con la agencia), sugeriría que tener un papel distintivo es crucial para responder al desafío de la suficiencia y al desafío de la distinción. Los papeles que la creencia y la percepción tienen en nuestras vidas psicológicas y teorías epistemológicas difieren y me gustaría sugerir que la mejor manera de explicar (A3) es una que se enfoca en estos distintos papeles.

Incluso si la creencia es como una experiencia perceptiva o una adivinación en cuanto a que son evaluables en términos de corrección y potencialmente en términos de su aptitud, la creencia difiere de estas cosas en cuanto a que es una fuente potencial de razones especificadas proposicionalmente. Si hay algo que es la razón del sujeto para creer, sentir, o hacer, las razones especificadas proposicionalmente tienen que ser algo que el sujeto considera que es el caso. Esto se debe a que la especificación de las razones del sujeto captura a la luz de lo cual el sujeto cree, siente o actúa, algo que captura la cosa o las cosas que, desde la perspectiva del sujeto, hicieron a la creencia, la emoción o la acción buenas, adecuadas, apropiadas, etc. Las razones del sujeto para creer, sentir, o pensar no podrían ser que la respuesta relevante tenía una característica F si el sujeto no creyese que la cosa tiene esta característica. Los estados o eventos no-dóxicos tales como las experiencias o las apariencias difieren de la creencia precisamente en que no involucran un compromiso por parte del sujeto con la verdad del contenido relevante y, por 
lo tanto, tales contenidos (si es que no son también adoptados porque son creídos) no podrían especificar las razones del sujeto.

Una adivinación puede motivar. Si tú adivinas que la manera en la que puedes llegar a un lugar seguro es trepando un árbol, podrías trepar un árbol en consecuencia. Una adivinación, sin embargo, no te puede dar una razón que motiva de la misma manera que lo puede hacer una creencia. De la misma manera que una adivinación, una creencia puede sacarte del agua, pero no sales del lago por la ražón de que un cisne enojado va en tu dirección si sólo adivinas que esto es el caso, pero no lo crees. Al adivinar, no tienes que considerar a $p$ como verdadera, pero $p$ no puede ser tu razón para hacer $\Phi$ a menos que lo tomes como verdadero. Al adivinar, probablemente no estás no-accidentalmente en lo correcto sobre si $p$, pero tal conexión no-accidental con el hecho es un requisito para que puedas ser guiado por éste.

No es sólo una cuestión de qué haces, también importa por qué haces lo que haces. Para castigar a alguien por una fechoría, tienes que asumir que son responsables por la fechoría en cuestión. Si adivinas que alguien fue responsable e impones una sanción o un castigo, esto sería objetable por esta misma razón. Quizás Austin estaba en lo correcto al señalar que los amantes no besan a sus queridas con un ojo puesto en el bien común, y quizás también estaba en lo correcto al señalar que los utilitaristas no deberían tener problemas con estos besos, pero las queridas deberían objetar si sus amantes las besan sólo porque los amantes adivinaron que serían sus queridas las que recibirían sus besos.

De hecho, podemos usar la noción de creencia apta para proporcionar una elucidación de las razones que motivan potenciales, cosas que podrían ser las razones del sujeto para creer cosas, sentir cosas, o hacer cosas sin tener que cambiar lo que piensa con respecto a la proposición relevante. Puesto que para que un sujeto haga $\Phi$ por la razón de que $p$ o que la razón del sujeto para hacer $\Phi$ sea que $p$, tiene que ser el caso que $p$. También tiene que ser el caso que el sujeto considere que $p$ es el caso. La creencia apta involucra una creencia correcta así que la corrección y considerar que algo es el caso está construido dentro de la noción. Para que la razón de un sujeto para hacer $\Phi$ haya sido que $p$, el sujeto debería ser guiado por el hecho de que $p$. Esta guía requiere de una conexión no-accidental. La creencia apta proporciona esto. Un sujeto puede hacer $\Phi$ por la razón de que $p$ si y sólo si cree aptamente que $p$, y esto explica (A3). ${ }^{14}$ Dado que la creencia de un sujeto es

${ }^{14}$ Esta segunda postura está disponible para alguien que ofrece el primer tipo de respuesta al problema de la distinción, pero no es claro que esta respuesta requiera que nosotros aceptemos algún supuesto controvertido particular sobre la relación entre la creencia y la agencia, no a 
apta si y sólo si constituye conocimiento, podemos ahora explicar algunas de las intuiciones cruciales que prestan apoyo a (A2).

Notemos que cuando desarrollamos esta postura para distinguir a las creencias de las adivinaciones y las percepciones, es bastante tentador decir que el punto o el propósito de las creencias es hacer algo que la percepción y la adivinación no pueden hacer, a saber, poner al sujeto en una posición de ser guiados por hechos que considera que ocurren. He sugerido (y argumentado en otro lugar) que esto requiere de más que la simple creencia correcta y la creencia diestra. Esto requiere de creencia apta, algo que yo considero conocimiento. Quizás, entonces, deberíamos considerar la posibilidad de que la meta de la creencia no sea sólo la corrección. ${ }^{15}$ Quizás la meta o el propósito de la creencia sea ser conocimiento. Sólo las creencias que constituyen conocimiento pueden hacer lo que se supone que las creencias deben hacer si éstas nos van a proporcionar razones que motivan potenciales y tales razones están constituidas por hechos.

Si consideramos al problema de la distinción de esta manera y tratamos de explicar (A3) en términos del papel distintivo que tiene la creencia y, en consecuencia, apelamos a este papel para distinguir un tipo de actitud apta de otras, ¿dónde nos deja esto? Parece que la teoría del valor de la creencia requiere que importemos consideraciones más allá de aquellas que determinan si la actitud es apta y nos pide que apelemos a consideraciones adicionales acerca de la función o el papel que ocupa la creencia. ¿Sugiere esto que el marco de la actuación normativa está

menos de que haya algún argumento ulterior que muestre que la adquisición y posesión de razones que motivan potenciales requiera por sí mismo de algún tipo de control agentivo, del tipo que Sosa considera que está involucrado en la creencia.

${ }^{15}$ Esto parece concordar con el espíritu de la sugerencia de Sosa (2015) sobre las metas respectivas de la adivinación y de los juicios. Para una discusión útil de la relación entre la creencia y la adivinación, véase Carter (MS), Owens (2003) Sorensen (1984), y Walker (MS). Una división interesante en estas discusiones es aquella entre gente que cree que las adivinaciones no pueden resultar ser casos de conocimiento y aquellos que piensan que sí lo pueden ser. Si bien encuentro muchas de las observaciones de Sorensen sugerentes, no estoy completamente convencido de que no es posible tanto adivinar que $p$ y llegar a saber que $p$ debido a los casos que Sosa discute. Por un lado, parece que no hay irracionalidad de mi parte si habiendo expresado una adivinación diciendo, 'está lloviendo afuera' me apresuré a añadir que no puedo saber si esto es así, pero sería irracional creer completamente esto al mismo tiempo que reconozco que no sé si está lloviendo afuera. Esto sugiere que Sorensen quizás está en lo correcto al decir que uno no puede adivinar y saber. Por otro lado, creo que Sosa está en lo correcto en que su caso es uno en el que algo que se "siente" como una adivinación y sin embargo podría resultar ser conocimiento, o algo cercano a conocimiento. 
incompleto? Esto es poco claro. Por un lado, pareciera que el marco está incompleto en la medida que sugiere que la elucidación completa del valor de la creencia apta o del juicio requiere más que la aptitud y por lo tanto requiere que pensemos sobre algo más además de si la corrección es atribuible a la habilidad. Por el otro lado, pareciera que no lo es en la medida que no podemos evaluar realmente una actuación como el juicio sin tener una idea del sentido del punto o del propósito de éste. La lección que tomaría de esto, sin embargo, es que no tendremos una explicación completa de (A3) a menos que pensemos acerca del papel distintivo que tiene la creencia, un papel que, he sugerido, involucra proporcionarnos una aprehensión de la verdad de manera que estas verdades puedan servir como nuestras razones para pensar, sentir, y hacer cosas. ${ }^{16}$

\section{TIPOS DE BONDAD}

Una dificultad que enfrentamos cuando intentamos abarcar la literatura sobre el valor epistémico y las intuiciones que motivan las discusiones es que no es para nada claro qué tipo de bien o valor es el que les interesa a quienes discuten. En algunos lugares, parece que el veritismo se entiende como la postura de que la creencia verdadera es buena simpliciter, un portador de bondad intrínseco. El veritismo no tiene que interpretarse de esta manera porque el habla de "bien" puede ser tomada de distintas maneras. Tal manera de hablar, por ejemplo, podría tomarse como habla sobre lo que es bueno para un pensador, algo que tiene que ver con qué tan bien van las cosas para este pensador.

\footnotetext{
${ }^{16}$ Se pueden encontrar argumentos adicionales a favor de la idea de que la creencia es distintiva en tanto que ocupa este papel y que la percepción no nos pone por sí misma en la posición de pensar, sentir o hacer cosas con base en razones especificadas proposicionalmente en Littlejohn (de próxima aparición, b y c). Al adoptar esta postura, tenemos que rechazar la postura, defendida por McDowell (1994) y por la ola reciente de los teóricos de la "prioridad de las razones" (e.g., Schroeder (2015)) que piensan que tanto la percepción como la creencia podrían permitir a un sujeto no sólo hacer $\Phi$, sino que le permiten hacerlo de manera tal que su razón para hacer $\Phi$ es que $p$. Podemos ver que tales posturas se enfrentan a problemas por dos razones. La primera, como nota Unger (1975), es que todas las adscripciones de razones especificadas proposicionalmente implican adscripciones de conocimiento, pero es crucial para estas posturas que tratan a la percepción como una fuente potencial de razones que sea posible percibir sin saber. En segundo lugar, es bastante claro que si alguien está molesto por $p$ no estaría molesto por $p$ si sus experiencias hubiesen sido las mismas, pero si no creyera que $p$. Si la experiencia no puede tener este papel de proporcionar razones, no hay misterio en absoluto.
} 
No creo que el veritismo sea una postura muy plausible si se la entiende como una afirmación acerca de los tipos de cosas que son buenas simpliciter para un pensador. Los casos considerados antes causan dificultades para el veritismo en cualquier lectura, pero hay problemas adicionales con estas ideas. Para empezar, simplemente soy incapaz de ver por qué dos mundos podrían diferir en sus valores si fuesen tan similares como es posible con excepción del hecho de uno contiene una creencia verdadera adicional que el otro carece. Por otro lado, no logro ver cómo es que dos vidas difirieran en términos de qué tan buenas son si sólo difiriesen de esta manera.

Cuando hablamos sobre el bien, no tenemos que tomar esto como una manera de hablar sobre las cosas que son buenas simpliciter o de las cosas que son buenas para un pensador y que hacen una diferencia con respecto al bienestar. Algunas maneras de hablar sobre lo bueno deberían entenderse como habla sobre la bondad atributiva. Algunas deberían ser entendidas como habla sobre la bondad normativa. Si estamos interesados en la noción de bondad atributiva, éste es el tipo de bien que está en cuestión cuando hablamos de buenas tostadoras, buenos asesinos o buenos argumentos. Son ejemplos de una clase que tienen las características que los vuelven buenos de manera relativa a ciertos propósitos. Si estamos interesados en la bondad normativa, este es el tipo de bien que está en cuestión cuando hablamos de cosas que son buenas porque son apropiadas. En algunas teorías sobre mantener promesas, es bueno mantener una promesa porque es correcto hacerlo; no es que sea correcto porque mantener la promesa promueva alguna noción independiente de bondad.

Tampoco creo que el veritismo sea una propuesta bastante plausible sobre estos tipos de bienes. Supongamos que Agnes cree irracionalmente que hay una conspiración en su contra y supongamos que su creencia es correcta. No creo que haya algún sentido en el que la creencia de Agnes de que cree irracionalmente que hay una conspiración en su contra vaya en contra de su propio bien. No es una creencia apropiada y tales creencias irracionales no pueden hacer lo que las creencias se supone que deben hacer (i.e., mantenernos en contacto con la realidad). El gnosticismo aquí parece ser una mejor opción. Es plausible que cualquier creencia que constituye conocimiento hace lo que las creencias se supone que deben hacer y deberían contar como bienes atributivos. También es plausible que cualquier creencia de este tipo se tiene de manera apropiada y que es buena por esa razón.

Una vez que vemos esto, deberíamos ver por qué las normas epistémicas no tienen que ver con maximizar los bienes epistémicos. También podemos dar sentido a una de las observaciones de Sosa sobre la bondad epistémica y el valor, una que recibe muy poca atención en las discusiones: 
Paradójicamente, uno puede ser un crítico experto dentro de ... un dominio al mismo tiempo que considera que no hay un valor transcendente a ese dominio. De esta manera, alguien que tiene conocimiento acerca de las armas y su uso para cazar, para fines militares, etcétera podría sufrir una conversión que hace el uso de armas detestable para ellos. El disparo bueno es de esta manera agotado de cualquier valor real que pueda discernir en éste. Sin embargo, su juicio crítico dentro de ese dominio puede superar el de cualquier otra persona, así sea un amante de las armas o no. Los dominios críticos pueden ser vistos como aislados, de maneras que nuestro ejemplo sugiere (2007: 74).

No queremos leer el veritismo o el gnosticismo como afirmaciones sobre tipos de valores acerca de los cuales no podemos tomar esta actitud independiente de manera que sería imposible que nuestras evaluaciones estuviesen aisladas de estas maneras. Cuando juzgamos que algo es bueno en términos atributivos o normativas, podemos tomar estas actitudes independientes, asumiendo que no estamos racionalmente obligados a preocuparnos sobre si los buenos elementos relevantes pueden llevar a cabo la función especificada o si alguien se ajusta a las normas relevantes. (Aquí es útil pensar sobre las normas de etiqueta o de la ley, normas a las que una persona puede ser racionalmente indiferente). ${ }^{17}$

Si hay algo correcto sobre esta línea de pensamiento, entonces el trabajo de nuestra teoría del valor epistémico no es identificar algún bien o valor que haya que resaltar y que después haya que utilizar para evaluar a las normas candidatas. En su lugar, nuestra teoría del bien epistémico podría simplemente registrar nuestras posturas sobre qué tipos de creencias llevan su función a cabo de manera correcta o son responsivas a estándares normativos. Una vez que esto se ha hecho explícito, es fácil ver que las preocupaciones sobre el consecuencialismo simplemente nunca despegan del piso. ${ }^{18}$ Nuestra teoría de la creencia diestra nos permite ver que la creencia diestra es diestra por cómo es ésta, no pensando sobre su papel general al contribuir causalmente a estados de cosas epistémicamente buenos.

\footnotetext{
${ }^{17}$ Podría ser que hay diferencias importantes entre las normas epistémicas y las normas legales precisamente porque pareciera que tienen diferentes implicaciones para la racionalidad. El punto que quiero señalar aquí es que las diferencias no van a ser explicadas apelando a las afirmaciones sobre los que es bueno simpliciter o sobre los que es bueno para un pensador. Pueden encontrarse discusiones útiles sobre de dónde podrían (y no podrían) obtener las normas epistémicas su fuerza en Cote-Bouchard (de próxima aparición) y Maguire y Woods (MS). 18 Puede encontrarse una discusión crítica de las posturas consecuencialistas a las normas epistémicas, véase Berker (2013), Firth (1982), Jenkins (1997), y Littlejohn (2012, 2016).
} 


\section{DESTREZA}

Cuando una actuación es diestra, esto tiene que ver con el papel de algunas competencias que residen en el agente. Si pensamos en la meta de la creencia como si tuviera que ver con la verdad, es tentador pensar que una creencia es diestra porque es el resultado del ejercicio de una competencia que produce de manera fiable creencias verdaderas (en circunstancias apropiadas) (Sosa 2009: 38). Hay algunas razones para pensar que deberíamos resistir la tentación de pensar sobre la destreza de esta manera, particularmente si estamos preocupados sobre las loterías y casos similares. Dado que los lectores quizás están cansados de los casos de lotería, me voy a enfocar en un tipo de caso similar:

Prisioneros. Cien prisioneros están haciendo ejercicio en el patio de la prisión. Noventa y nueve de ellos participan de manera repentina en un ataque planeado contra un guardia; el prisionero número cien no toma parte en el ataque. No hay evidencia [adicional] disponible para demostrar quién participó en el ataque y quién no.

Sabiendo lo que sabemos, deberíamos tener un alto grado de credibilidad de que cualquier prisionero del patio seleccionado al azar participó en el ataque, pero la pregunta no es si deberíamos de tener este grado alto de credibilidad sino qué nos dice esto sobre la creencia, el juicio, el castigo, la culpa y, en consecuencia, de la creencia diestra.

Muchos sienten de manera bastante fuerte que no sería apropiado castigar a un prisionero de este grupo seleccionado al azar. Esto no tiene que ver (necesariamente) con que estas personas sean "blandas con el crimen" sino porque creen que no deberíamos castigar a los culpables por sus crímenes a menos que su culpa esté más allá de la duda razonable. No creo que las dudas se vuelvan no razonables si incrementamos el grupo en el patio de la prisión de manera que haya 1000 prisioneros y 999 atacantes.

Si caracterizamos a la creencia justificada o a la creencia racional como creencia diestra y caracterizamos esto, posteriormente, de acuerdo con las propuestas fiabilistas, es difícil ver cómo podríamos evadir el resultado de que alguien que está en un jurado podría juzgar de manera justificada que un prisionero elegido al azar estuvo involucrado en el ataque. La creencia, si estuviese justificada, racionalizaría la culpa y las actitudes reactivas que también estarían a su vez justificadas. Así, sería difícil resistir la conclusión de que el castigo también estaría justificado. (Si de algo ayuda, podríamos enfocarnos primero en el caso en el que el acusado estuvo involucrado). Hablando por mí mismo, no puedo sino pensar que algo ha salido mal. Parece incorrecto castigar, incorrecto culpar, y parece que estos hechos son 
evidencia bastante sólida de que sería incorrecto juzgar que el prisionero estuvo involucrado en el ataque.

Una manera en la que podemos presionar la intuición aquí sería notando que ciertamente sería incorrecto castigar a 100 prisioneros en el caso descrito. ${ }^{19}$ Las bases para juzgar que algún prisionero en particular estuvo involucrado, sin embargo, están presentes en cada caso. Si no es inapropiado juzgar que algún prisionero estuvo involucrado y que merece un castigo, parece razonable condenar y castigar, de modo tal que el hecho de que no sería razonable emitir 100 condenas debería preocupar a aquellos que piensan que esto podría ser resuelto por las probabilidades y los valores que damos a las sentencias de culpable y a las sentencias de inocente.

Hay una manera sencilla de vindicar estas intuiciones y varias maneras difíciles de hacerlo. ${ }^{20}$ Comencemos con la manera sencilla. Supongamos que pensamos que la creencia y el juicio tienen como meta el conocimiento, no sólo la corrección o la verdad. Supongamos, además, que podemos saber a priori que los juicios que se basan en probabilidades en casos como éste no pueden constituir conocimiento. Si caracterizamos la creencia diestra como una creencia que es producida mediante una competencia que produce de manera fiable conocimiento, no meramente creencia verdadera, en circunstancias adecuadas, la creencia en la culpa del acusado no sería diestra, no podría ser apta, y no tendríamos razón para pensar que las actitudes reactivas subsecuentes ni que las sentencias estarían justificadas. (Si tu creencia en $p$ no está justificada y 'racionaliza" alguna actitud reactiva, muy probablemente ésta tampoco estará justificada). Dado que no podríamos estar justificados en juzgar que debemos castigar en un caso ni podríamos estar justificados en castigar en este caso, la preocupación sobre si castigar o no a los 100 sujetos no surge.

\footnotetext{
${ }^{19}$ Agradezco a Peter Dennis que me haya señalado este punto. Puede encontrarse una discusión útil de un acercamiento alternativo a estas cuestiones en Steele (MS). Blome-Tillman (2015) ofrece una propuesta de conocimiento primero atractiva con respecto a la evidencia en juicios civiles.

${ }^{20}$ Si bien defiendo la postura de que una creencia justificada es una creencia apta (i.e., una que constituye conocimiento porque es correcta debido a que es diestra), la mayoría de los epistemólogos que desarrollan una teoría de la creencia diestra que sigue estas líneas quieren adoptar posturas en las que puede haber creencias falsas justificadas siempre y cuando las creencias falsas sean tales que pueden haber sido, de alguna manera, buenos candidatos para ser conocimiento. Véase Bird (2007), Miracchi (2015), y Kelp (de próxima aparición), donde pueden encontrarse distintas maneras de desarrollar estas posturas. En (2012), argumenté que estas posturas cometen el error de tomar la excelencia racional como la marca de la justificación cuando de hecho tal excelencia se requiere para una excusa, pero este desacuerdo no importa dados nuestros propósitos en este artículo.
} 
Si los lectores quieren vindicar las intuiciones relevantes sin tratar al conocimiento como la meta de la creencia plena y el juicio, y caracterizar a la creencia diestra en términos de competencias que generan conocimiento en circunstancias apropiadas, hay maneras de tratar de vindicar las intuiciones sobre la culpa, el castigo, etcétera, pero son muy complicadas y difíciles de defender. Quizás la manera más simple de ver el problema que enfrentarías consiste en considerar esta observación de Sophie Horowitz:

... un agente racional debería hacer el bien según su propio criterio, de una manera particular: a grandes rasgos, debería seguir una regla epistémica que toma racionalmente como la que es más conducente a la verdad. Sería irracional, según este pensamiento, considerar que alguna regla epistémica es más conducente a la verdad que la propia, pero no adoptarla (Horowitz 2014: 43).

Si la meta de la creencia es sólo la verdad y consideramos una regla que, digamos, prohíbe la creencia en casos como Prisioneros, no es difícil imaginar una regla rival que sea más permisiva y que permita la creencia en tales casos y que no sea difícil de ver cómo creer de acuerdo con la regla más permisiva sería más conducente a la verdad. Para prohibir la creencia en casos como Prisioneros, entonces, uno tendría que introducir algún obstáculo que forzara seguir una regla conducente a la verdad que maximizara los bienes epistémicos esperados o bien adoptar una actitud hacia los valores de la creencia verdadera y falsa que justificase la aversión al riesgo en el rango relevante de casos. Parece que la primera opción llevaría a restricciones más bien arbitrarias sobre si es apropiado adoptar creencias. La segunda opción requiere una aversión al error que parece más bien patológica, en particular cuando reflexionamos sobre nuestros propios hábitos de confiar en fuentes testimoniales que, cuando reflexionamos al respecto, parecen ser menos fiables que los juicios que formamos en casos como Prisioneros.

Una vez que adoptamos una postura revisada de la competencia y de la creencia diestra, una que caracteriza al conocimiento como la meta de la creencia y del juicio, encontramos soluciones naturales a casos que de otro modo parecerían difíciles de resolver. Mocionaré brevemente tres problemas que podríamos disolver fácilmente modificando nuestra concepción de la creencia diestra. Primero, está el problema de hacer que nuestras intuiciones sobre las loterías concuerden con nuestras intuiciones cobre los casos prefacio. Desde la perspectiva de una teoría veritista de las normas epistémicas, una que se preocupa principalmente por la adquisición de creencias verdaderas y evitar las creencias falsas, es increíblemente difícil proporcionar una teoría en la que (a) sea inapropiado creer proposiciones 
de lotería, pero (b) que sea apropiado creer cada afirmación en un libro que tiene la aseveración del prefacio (i.e., la aseveración de que el libro contiene un error). Para dar una explicación correcta de la primera intuición, necesitamos que ciertas restricciones arbitrarias que conocemos a priori se pongan en el medio de la maximización esperada de bienes epistémicos (tal y como el veritista concibe éstos) o bien tenemos que pensar que la creencia falsa es un mal epistémico abominable, uno que justificaría niveles patológicos de aversión al riesgo. Si hacemos cualquiera de estos movimientos con la finalidad de vindicar las intuiciones a favor de (a), se vuelve casi imposible vindicar las intuiciones a favor de (b). Si, sin embargo, adoptamos una postura gnóstica a las normas epistémicas, la asimetría se puede explicar fácilmente. Cada afirmación en un libro que se hizo mediante una investigación cuidadosa podría ser potencialmente un caso de conocimiento incluso si el conjunto de creencias es inconsistente. Así, las afirmaciones en el libro podrían todas emparejarse con creencias diestras, pero ninguna de las creencias en el caso de la lotería podría ser un caso potencial de conocimiento. ${ }^{21}$

En segundo lugar, tenemos el problema presentado por los absurdos mooreanos tales como "el acusado es culpable, pero no sé si lo es". Supongamos que estoy en lo correcto y que no podemos saber en Prisioneros que alguien elegido al azar estuvo involucrado en el ataque. Si esto es así, deberías tener esencialmente el mismo grado de credibilidad en esta conjunción que el grado de credibilidad que tenemos en el primer conyunto. (Esto es así porque formamos la conjunción combinando una verdad a priori sobre el primer conyunto). Desde el punto de vista del veritista, entonces, no haríamos un buen trabajo maximizando la bondad epistémica esperada si no creyésemos tales cosas. Desde el punto de vista gnóstico, sin embargo, es obvio por qué no deberíamos creer tales cosas. La conjunción no es un objeto potencial de conocimiento y sabemos esto a priori. De esta manera, no hay nada que debería producir una creencia.

Tercero, pensemos acerca de la dificultad que enfrentamos al entender las conexiones descriptivas y normativas entre la credibilidad y la creencia plena. Notemos que hay algo que presta apoyo a la adivinación y algo que presta apoyo a tener una credibilidad alta en casos como Prisioneros. Esto proporciona evidencia para creer que tales cosas están bajo diferentes presiones racionales que la creencia plena. Una vez que vemos a la creencia plena y a la credibilidad como si estuviesen bajo diferentes presiones racionales, deberíamos de ver que hay pocas razones para adoptar el tipo de postura lockeana que considera a la creencia plena racional

${ }^{21}$ Este juicio no concuerda con el tratamiento de Sosa (2015) del caso de la lotería. 
como meramente un caso de credibilidad racional. ${ }^{22}$ Si pensamos en la credibilidad como si rastrease la fuerza del soporte evidencial, podemos verla como si racionalizase la creencia en los casos en los que el sujeto no está en posición de ver que no podría saber y como racionalizando una adivinación (pero no una creencia) en los casos en los que el sujeto reconoce que no está en una posición de saber. ${ }^{23}$ No hay problema en identificar un umbral de credibilidad lo suficientemente fuerte porque cuando el sujeto reconoce que no está en posición de saber si $p$, reconoce que no puede hacer un juicio sobre si $p$, ni siquiera si su credibilidad racional se vuelve arbitrariamente fuerte.

\section{CONCLUSIÓN}

De una manera indirecta, he tratado de defender una posición sobre la relación entre la creencia y el conocimiento que podría estar en conformidad con la postura de Sosa, dada su reciente discusión de la relación entre el juicio y la adivinación. Al juzgar, sugiere, buscamos la aptitud, no la mera precisión. Al adivinar, en contraste, piensa que podríamos sólo buscar la corrección. Esta postura, si es tomada con seriedad, podría forzarnos a repensar los supuestos veritistas que son prominentes en la literatura. De igual manera, esto nos podría forzar a revisar nuestras teorías de creencia diestra o racional de maneras que ciertos casos que parecían inicialmente complicados no parecen ser difíciles de entender. Esto podría requerir revisiones a la teoría del valor que se presentó inicialmente en $\$ 2$, pero no creo que sean el tipo de cambios que serían costosos para Sosa.

Traducción de Alfonso Anaya
Clayde Littlejobn
King's College London
cmlittlejohn@gmail.com

${ }^{22}$ En Littlejohn (2016), argumento que las presiones racionales que deberían llevarnos a suspender el juicio sobre si $p$ no lo hacen automáticamente dándonos una razón para mermar nuestra confianza en $p$. El reconocimiento de que no estás en una posición de saber si $p$ por ejemplo, te da una razón decisiva para suspender el juicio pero no debe de darte, de esta manera, ninguna razón para mermar tu confianza.

23 Puede encontrarse una discusión interesante de la relación entre la credibilidad y la adivinación en Horowitz (de próxima aparición). 


\section{BIBLIOGRAFÍA}

Ahlstrom-Vij, K., and Dunn, J. (2014), "A Defence of Epistemic Consequentialism”, The Philosophical Quarterly 64: 541-51.

Álvarez, M. (2010), Kinds of Reasons. Oxford University Press.

Benton, M., and Turri, J. (2014), "Iffy Predictions and Proper Expectations", Synthese 191: 1857-66.

Berker, S. (2013), “The Rejection of Epistemic Consequentialism”, Philosopbical Issues 23: 363-387.

BIRD, A. (2007), "Justified Judging", Philosophy and Phenomenological Research 74: 81-110.

Blome-Tillman, M. (2015), "Sensitivity, Causality, and Statistical Evidence in Courts of Law", Thought 4: 102-12.

BRADFord, G. (2015), Achievements, Oxford University Press.

Brewer, B. (2011), Perception and its Objects, Oxford University Press.

BYRne, A. (2009), "Experience and Content", The Philosophical Quarterly 59: 429-52.

CARTER, J. A. (MS), Sosa on Knowledge, Judgment, and Guessing.

Comesana, J., and McGrath, M. (2014), "Having False Reasons", en C. Littlejohn and J. Turri (ed.), Epistemic Norms. Oxford University Press, pp. 59-79.

Cote-Bouchard, Ch. (Forthcoming), "Can the Aim of Belief Ground Epistemic Normativity?” Philosophical Studies.

Dutant, J. (2012), "The Value and Expected Value of Knowledge”, Dialogue 51: 141-62.

FIRTH, R. (1981), "Epistemic Merit, Intrinsic and Instrumental", Proceedings and Addresses of the American Philosophical Association 55: 5-23.

Goldman, A. (1999), Knowledge in a Social World, Oxford University Press.

HACKER, P.M.S. (2013), The Intellectual Powers, Wiley.

Ho, Tsung-Hsing, (Forthcoming), "Epistemic Normativity as Performance Normativity", Theoria.

HorowiTZ, S. (2014), "Immoderately Rational”, Philosopbical Studies 167: 41-56.

— (Forthcoming), "Accuracy and Educated Guesses", Oxford Studies in Epistemology.

Huemer, M. (2007), "Compassionate Phenomenal Conservatism", Philosophy and Phenomenological Research 74: 30-55.

HyMan, J. (2015), Action, Knowledge, and the Will. Oxford University Press.

JENKINS, C. (2007), "Entitlement and Rationality", Synthese 157: 25-45.

KelP, C. (Forthcoming) "Justified Belief: Knowledge-First Style", Philosopby and Phenomenological Research.

LitTlejohn, C. (2012), Justification and the Truth-Connection. Cambridge University Press.

— (2014), "Fake Barns and False Dilemmas". Episteme 11: 369-89.

- (2016), "Who Cares What You Accurately Believe?", Philosopbical Perspectives 29: 217-48.

- (Forthcoming), "The Good in the Right", en J. Dunn and K. Ahlstrom-Vij (ed.), Epistemic Consequentialism. Oxford University Press.

- (Forthcoming, b.), "Evidence and its Limits", en C. McHugh, J. Way, and D. Whiting (ed.), Normativity: Epistemic and Practical. Oxford University Press. 
LitTLejohn, C. (Forthcoming, c.), "How and Why Knowledge is First", en A. Carter, E.

Gordon, and B. Jarvis (ed.), Knowledge-First Epistemology. Oxford University Press.

Logue, H. (2014), "Experiential Content and Naive Realism: A Reconciliation”, en B.

Brogaard (ed.), Does Perception Have Content? Oxford University Press, pp. 220-42.

LYNCH, M. (2004), True to Life. MIT University Press.

MCDowell, J. (1994), Mind and World. Harvard University Press.

MAGUIRE, B., and JACK W. (MS), Explaining Epistemic Normativity.

Miracchi, L. (2015) “Knowledge is All You Need”, Philosophical Issues 25: 353-78.

Owens, D. (2003). "Does Belief Have an Aim?”, Philosophical Studies 115: 283-305.

Pritchard, D. (Forthcoming), "Veritism and Epistemic Value", en H. Kornblith and B.

McLaughlin (ed.), Alvin Goldman and his Critics. Wiley.

Redmayne, M. (2008), "Exploring the Proof Paradoxes", Legal Theory 14: 281-309.

ReIlland, I. (2015), "Experience, Seemings, and Evidence", Pacific Philosophical Quarterly 96: 510-34.

SARTWELL, C. (1992), "Why Knowledge is Merely True Belief”, Journal of Philosophy 89: 167-80.

SCHELlEnBERG, S. (2014), "The Relational and Representational Character of Perceptual

Experience", en B. Brogaard (ed.), Does Perception Have Content? Oxford University

Press, pp. 199-220.

Schroeder, M. (2015), "Knowledge is Belief for Sufficient (Objective and Subjective)

Reason", en J. Hawthorne and T. Gendler (ed.), Oxford Studies in Epistemology 5.

Oxford University Press, pp. 226-52.

SIEGEL, S. (2011), The Contents of Visual Experience. Oxford University Press.

Sorensen, R. (1984), "Unique Alternative Guessing”, Logique et Analyse 27: 77-85.

SosA, E. (2001), For the Love of Truth. In L. Zagzebski and A. Fairweather (ed.) Virtue

Epistemology: Essays on Epistemic Virtue and Responsibility. Oxford University Press, pp. 49-62.

- (2007), A Virtue Epistemology Vol. I. Oxford University Press.

- (2013), "Epistemic Agency", The Journal of Philosophy 110: 585-605.

- (2015), Judgment and Agency. Oxford University Press.

STEELE, K. (MS), Crime, Punishment, and 'Individualized' Evidence.

SyLVAN, K. (2013), "Truth Monism without Teleology", Thought 1: 161-9.

Thomson, J. (2008), Normativity. Open Court.

Travis, Ch. (2004), "Silence of the Senses". Mind 113: 57-94.

Tucker, C. (2010), "Why Open-Minded People Should Endorse Dogmatism”, Philosophical Perspectives 24: 529-45.

Unger, P. (1975), Ignorance. Oxford University Press.

WALKER, B. (MS), Enquiry and the Value of Knowledge.

Williamson, T. (2000), Knowledge and its Limits. Oxford University Press.

ZagzeBKSI, L. (1996), Virtues of the Mind: An Inquiry into the Nature of Virtue and the Ethical Foundations of Knowledge. Cambridge University Press. 